\title{
Pattern Formation in Tri-Trophic Ratio-Dependent Food Chain Model
}

\author{
Dawit Melese, Sunita Gakkhar \\ Department of Mathematics, Indian Institute of Technology Roorkee, Roorkee, India \\ E-mail: mahifikir@gmail.com, sungkfma@iitr.ernet.in \\ Received October 27, 2011; revised November 26, 2011; accepted December 5, 2011
}

\begin{abstract}
In this paper, a spatial tri-trophic food chain model with ratio-dependent Michaelis-Menten type functional response under homogeneous Neumann boundary conditions is studied. Conditions for Hopf and Turing bifurcation are derived. Sufficient conditions for the emergence of spatial patterns are obtained. The results of numerical simulations reveal the formation of labyrinth patterns and the coexistence of spotted and stripelike patterns.
\end{abstract}

Keywords: Reaction-Diffusion Equations, Hopf Bifurcation, Turing Instability, Turing Pattern, Food Chain

\section{Introduction}

Food chains in the environment are very important systems in ecological science, applied mathematics, economic and engineering science [1]. They have been applied to management in Aquatic ecosystem for problems like water quality and lake management [2]. Modeling of food chain dynamics has challenges in the fields of both theoretical ecology and applied mathematics. Tri-trophic food chain models have been studied in both spatially homogeneous [1-4] and spatially inhomogeneous environments [5-10] for the last two decades. Those models exhibit rich and complex dynamics and nonlinear mathematical behavior, including varying numbers and stability of equilibrium states, limit cycles, patterns and chaos.

It is well known that in real life, the resources are not uniformly distributed in the habitat. The biological species move (diffuse) from place to place in search of food in their habitat and hence interact with each other and with the environment. This movement (diffusiveness) of species has an impact on their trophic interactions. Consequently, spatial patterns evolve.

One can easily observe patterns in both aquatic and terrestrial populations [10]. A number of recent contributions have begun to suggest that it is critical to begin to fully consider the implications of spatial flows on the dynamics of ecological communities [7]. In the literatures $[11,12]$, the spatial component of ecological interactions (trophic-interactions) is identified as an important factor in shaping ecological communities. However, there is lack of recognition about the role of spatial considera- tions in food chain dynamics [7]. In addition to this, ignorance of spatial scales of food chains limits our ability to predict how trophic interactions will vary in different contexts, across space and time [2]. The dynamics and stability of the interacting species in relation to spatial phenomena such as pattern formation has recently become a focus of intensive research in theoretical ecology [13], chemical and biological systems [14]. The spatial patterns modify the temporal dynamics at a range of spatial scales, whose effects must be incorporated in temporal ecological models that do not represent space explicitly. In recent decades, stationary patterns induced by diffusion have been studied extensively, and lots of important phenomena have been observed.

Spatial patterns of interacting species are ubiquitous in nature and may occur due to stochastic process, environmental fluctuations or variability, or deterministic process, growth and movement of interacting species. The deterministic process is intrinsic to the interacting species and results in population-driven and self-organized spatial patterns. The formation of population-driven spatial patterns was first pointed out by Alan Turing. Turing, one of the key scientist of the $20^{\text {th }}$ century, mathematically showed that a system of coupled reaction-diffusion equations can give rise to spatial concentration patterns of a fixed characteristic length from an arbitrary initial configuration due to diffusion-driven instability or Turing instability [15].

Reaction-Diffusion systems are capable of qualitatively imitating many biological patterns such as the stripes of a zebra, tiger and snakes or spots of a cheetah and 
even more irregular patterns such as those on leopards and giraffes, the patterns on exotic fish, butterflies or beetles through Turing instability.

Pattern formation for two species model [11-14,16-19] based on coupled reaction diffusion equations has been intensively investigated. The necessary and sufficient condition for Turing instability, which leads to the formation of spatial patterns, has been derived $[9,16,17,20]$ and very interesting patterns are also obtained from the numerical simulation results $[9,21,22]$.

For three species case, the authors [2,23] used a three species interacting discrete model to study the formation of pattern. In the literatures $[9,10,24]$, the authors have considered a food chain model with diffusion and investigate the persistence of the system, the stability of the positive steady state solution of the system.

In this paper, the formation of patterns in a tri-trophic food chain model with ratio-dependent Michaelis-Menten type functional response and diffusion has been investigated.

The organization of the paper is as follows: In Section 2 the mathematical model is given. Section 3 is devoted to the stability and bifurcation analysis of the system. Section 4 presents the results of numerical simulations. Section 5 is devoted to some conclusions.

\section{The Mathematical Model}

Let $U(X, Y, T), V(X, Y, T)$ and $W(X, Y, T)$ denote the densities of the prey, intermediate predator and top predator respectively at time $T$ and position $(X, Y)$ in the habitat $\Omega \subseteq \mathbb{R}_{+}^{2}$. The prey is assumed to grow logistically. The intermediate predator, $V$, and the top predator, $W$, follow the ratio-dependent Michaelis-Menten type functional response. Thus the mathematical model governing the spatiotemporal dynamics of the three interacting species prey-predator community can be described by the following system of reaction-diffusion equations.

$$
\begin{aligned}
& \left(\frac{\partial U}{\partial T}-D_{1}\left(\frac{\partial^{2} U}{\partial X^{2}}+\frac{\partial^{2} U}{\partial Y^{2}}\right)=r\left(1-\frac{U}{K}\right) U-\frac{B_{1} U V}{U+\beta_{1} V},\right. \\
& \left\{\begin{array}{l}
\frac{\partial V}{\partial T}-D_{2}\left(\frac{\partial^{2} V}{\partial X^{2}}+\frac{\partial^{2} V}{\partial Y^{2}}\right)=\frac{B_{2} U V}{U+\beta_{1} V}-\frac{B_{3} V W}{V+\beta_{2} W}-B_{4} V, \\
\quad(X, Y) \in \Omega, \\
\frac{\partial W}{\partial T}-D_{3}\left(\frac{\partial^{2} W}{\partial X^{2}}+\frac{\partial^{2} W}{\partial Y^{2}}\right)=\frac{B_{5} V W}{V+\beta_{2} W}-B_{6} W,(X, Y) \in \Omega,
\end{array}\right. \\
& \frac{\partial U}{\partial v}=\frac{\partial V}{\partial v}=\frac{\partial W}{\partial v}=0, \quad(X, Y) \in \partial \Omega, \\
& U(X, Y, 0)=U_{0}(X, Y) \geq 0, V(X, Y, 0)=V_{0}(X, Y) \geq 0 \text {, } \\
& W(X, Y, 0)=W_{0}(X, Y) \geq 0, \quad(X, Y) \in \Omega \text {. }
\end{aligned}
$$

The reaction parameters are assumed to be positive constants and have the usual biological meaning. The positive constants $D_{1}, D_{2}$ and $D_{3}$ are the diffusion coefficients of $U, V$ and $W$ respectively. $v$ is the outward unit normal vector to the smooth boundary $\partial \Omega$. The initial population densities $U_{0}(X, Y), V_{0}(X, Y)$ and $W_{0}(X, Y)$ are assumed to be positive and continuous functions.

Introduce the following non-dimensional variables and parameters so as to reduce the number of parameters of the system (2.1):

$$
\begin{aligned}
& u=\frac{U}{K}, v=\frac{\beta_{1} V}{K}, w=\frac{\beta_{1} \beta_{2} W}{K}, t=r T, \\
& x=X \sqrt{\frac{r}{D_{2}}}, y=Y \sqrt{\frac{r}{D_{2}}}, d_{1}=\frac{D_{1}}{D_{2}}, d_{3}=\frac{D_{3}}{D_{2}}, \\
& c_{1}=\frac{B_{1}}{r \beta_{1}}, c_{3}=\frac{B_{3}}{r \beta_{2}}, c_{i}=\frac{B_{i}}{r}(i=2,4,5,6) .
\end{aligned}
$$

The spatio-temporal system (2.1) is transformed to the following system of equations in non-dimensional variables:

$$
\begin{aligned}
& \left(\frac{\partial u}{\partial t}-d_{1}\left(\frac{\partial^{2} u}{\partial x^{2}}+\frac{\partial^{2} u}{\partial y^{2}}\right)=(1-u) u-\frac{c_{1} u v}{u+v},(x, y) \in \Omega,\right. \\
& \frac{\partial v}{\partial t}-\left(\frac{\partial^{2} v}{\partial x^{2}}+\frac{\partial^{2} v}{\partial y^{2}}\right)=\frac{c_{2} u v}{u+v}-\frac{c_{3} v w}{v+w}-c_{4} v,(x, y) \in \Omega, \\
& \left\{\frac{\partial w}{\partial t}-d_{3}\left(\frac{\partial^{2} w}{\partial x^{2}}+\frac{\partial^{2} w}{\partial y^{2}}\right)=\frac{c_{5} v w}{v+w}-c_{6} w,(x, y) \in \Omega,\right. \\
& \frac{\partial u}{\partial v}=\frac{\partial v}{\partial v}=\frac{\partial w}{\partial v}=0, \quad(x, y) \in \partial \Omega, \\
& u(x, y, 0)=u_{0}(x, y) \geq 0, v(x, y, 0)=v_{0}(x, y) \geq 0, \\
& w(x, y, 0)=w_{0}(x, y) \geq 0, \quad(x, y) \in \Omega \text {. }
\end{aligned}
$$

\section{Stability and Bifurcation Analysis}

The spatio-temporal system (2) has at most three spatially homogeneous non-negative steady states:

1) predators free steady state: $E_{0}=(1,0,0)$

2) top predator free steady state: $E_{1}=\left(u^{\prime}, v^{\prime}, 0\right)$ $u^{\prime}=1-c_{1}\left(1-\frac{c_{4}}{c_{2}}\right), v^{\prime}=\left(-1+\frac{c_{2}}{c_{4}}\right) u^{\prime}$,

3) coexistence of the three species: $E=(\tilde{u}, \tilde{v}, \tilde{w})$;

$$
\begin{aligned}
& \tilde{u}=\mathrm{c}_{1}\left(-1+\frac{1}{A}\right)+1, \tilde{v}=(A-1) \tilde{u}, \\
& \tilde{w}=\left(-1+\frac{c_{5}}{c_{6}}\right) \tilde{v}, \mathrm{~A}=\frac{\mathrm{C}_{2} C_{5}}{c_{3}\left(c_{5}-c_{6}\right)+c_{4} C_{5}} .
\end{aligned}
$$

The equilibrium point $E$ lies in the first octant if and 
only if

$$
C_{5}>C_{6}, A>1,0<c_{1}<\frac{A}{A-1}
$$

From biological point of view the stability of the nontrivial steady state $E$ which ensures the coexistence of the three species is of interest.

To perform linear stability analysis, the spatio-temporal system (2) is linearized at the spatially homogeneous steady state $E$ for small space and time dependent fluctuations. For this, set

$$
\begin{aligned}
& u(x, y, t)=\tilde{u}+\bar{u}(x, y, t) ;|\bar{u}(x, y, t)| \ll \tilde{u}, \\
& v(x, y, t)=\tilde{v}+\bar{v}(x, y, t) ;|\bar{v}(x, y, t)| \ll \tilde{v}, \\
& w(x, y, t)=\tilde{w}+\bar{w}(x, y, t) ;|\bar{w}(x, y, t)| \ll \tilde{w} .
\end{aligned}
$$

Let us assume solutions of the form

$$
\left(\begin{array}{l}
\bar{u}(x, y, t) \\
\bar{v}(x, y, t) \\
\bar{w}(x, y, t)
\end{array}\right)=\left(\begin{array}{l}
\alpha_{0} \\
\alpha_{1} \\
\alpha_{2}
\end{array}\right) e^{\lambda t} \cos \left(k_{x} x\right) \cos \left(k_{y} y\right),
$$

where $\lambda$ is the growth rate of perturbation in time $t$, $\alpha_{i}(i=0,1,2)$ represent the amplitudes, $k_{x}$ and $k_{y}$ are the wave number of the solutions. The corresponding linearized system has the characteristic equation

$$
\left|J-k^{2} D-\lambda I\right|=0 .
$$

Here $D=\operatorname{diag}\left(d_{1}, 1, d_{3}\right), \quad k^{2}=k_{x}^{2}+k_{y}^{2}$ and $J=\left(a_{i j}\right)_{3 \times 3}$ is the community matrix of the system (2). The elements are obtained as

$$
\begin{aligned}
& a_{11}=-1+c_{1}\left(1-\frac{1}{A^{2}}\right), a_{12}=-\frac{c_{1}}{A^{2}}, a_{13}=0, \\
& a_{21}=\frac{(-1+A)^{2} c_{2}}{A^{2}}, a_{22}=\left(\frac{c_{2}}{A^{2}}-c_{4}-c_{3}\left(1-\frac{c_{6}}{c_{5}}\right)^{2}\right), \\
& a_{23}=-\frac{c_{3} c_{6}^{2}}{c_{5}^{2}}, a_{31}=0, a_{32}=c_{5}\left(1-\frac{c_{6}}{c_{5}}\right)^{2}, a_{33}=c_{6}\left(-1+\frac{c_{6}}{c_{5}}\right) .
\end{aligned}
$$

The characteristic equation corresponding to $E$ is

$$
\lambda^{3}+b_{2}\left(k^{2}\right) \lambda^{2}+b_{1}\left(k^{2}\right) \lambda+b_{0}\left(k^{2}\right)=0,
$$

with

$$
\begin{aligned}
& b_{2}\left(k^{2}\right)=-\left(a_{11}+a_{22}+a_{33}-k^{2}\left(d_{1}+1+d_{3}\right)\right), \\
& b_{1}\left(k^{2}\right)=a_{11} a_{33}+a_{11} a_{22}+a_{22} a_{33}-a_{32} a_{23}-a_{21} a_{12} \\
& -k^{2}\left(\left(d_{1}+d_{3}\right) a_{22}+\left(1+d_{3}\right) a_{11}+\left(d_{1}+1\right) a_{33}\right), \\
& +k^{4}\left(d_{1}+d_{3}+d_{1} d_{3}\right)
\end{aligned}
$$

$$
\begin{aligned}
& b_{0}\left(k^{2}\right)=-a_{11} a_{33} a_{22}+a_{11} a_{32} a_{23}+a_{12} a_{21} a_{33} \\
& +k^{2}\left(\left(-a_{21} a_{12}+a_{11} a_{22}\right) d_{3}+\left(a_{22} a_{33}-a_{32} a_{23}\right) d_{1}+a_{11} a_{33}\right) \\
& -k^{4}\left(d_{1} a_{33}+a_{22} d_{3} d_{1}+a_{11} d_{3}\right)+k^{6} d_{1} d_{3} .
\end{aligned}
$$

The reaction-diffusion systems have led to the characterization of two basic types of symmetry-breaking bifurcations-Hopf and Turing bifurcation, responsible for the emergence of spatio-temporal patterns. See, for details, references [15,16,23,24].

According to Routh-Hurwitz criteria $\operatorname{Re}(\lambda(k))<0$ if and only if

$$
b_{0}\left(k^{2}\right)>0, b_{2}\left(k^{2}\right)>0, b_{1}\left(k^{2}\right) b_{2}\left(k^{2}\right)-b_{0}\left(k^{2}\right)>0
$$

Contradiction of any one of the above conditions implies the existence of an eigenvalue with positive real part, hence, instability. Turing instability (or diffusion driven instability) occurs if the homogeneous steady state $E$ is stable in the absence of diffusion $\left(k^{2}=0\right)$ but driven unstable by diffusion $\left(k^{2}>0\right)$. Thus we need two conditions which must hold simultaneously. First, the spatially uniform steady state must be stable to small perturbation, that is, all $\lambda\left(k^{2}\right)$ in Equation (5) have $\operatorname{Re}\left(\lambda\left(k^{2}=0\right)\right)<0$, and second, only patterns of a certain spatial extent, that is, patterns within a definite range of wave length $k$, can begin to grow, with $\operatorname{Re}\left(\lambda\left(k^{2} \neq 0\right)\right)>0$.

It is clear that the homogeneous steady state $E$ is locally asymptotically stable if and only if $b_{0}(0)>0$, $b_{2}(0)>0$ and $b_{1}(0) b_{2}(0)-b_{0}(0)>0$. But it will be driven unstable by diffusion if any of the conditions in Equation (3.4) fail to hold. However, it can be easily seen that diffusion driven instability cannot occur by contradicting $b_{2}\left(k^{2}\right)>0$. Hence, we have to look for conditions which reverse the sign of the other two conditions in Equation (3.4). The expressions for $b_{0}\left(k^{2}\right)$ and $b_{1}\left(k^{2}\right) b_{2}\left(k^{2}\right)-b_{0}\left(k^{2}\right)$ are both cubic functions of $k^{2}$ of the form:

$$
F\left(k^{2}\right)=F_{3} k^{6}+F_{2} k^{4}+F_{1} k^{2}+F_{0} ; F_{3}>0, F_{0}>0 .
$$

The coefficients $F_{i}(i=0,1,2,3)$ are given in Table 1.

For $F\left(k^{2}\right)$ to be negative for some positive real number $k^{2}(\neq 0)$, the minimum must be negative. This minimum occurs at

$$
k^{2}=k_{c}^{2}=\frac{-F_{2}+\sqrt{F_{2}^{2}-3 F_{1} F_{3}}}{3 F_{3}} .
$$

Now $k_{c}^{2}$ is real and positive if

$$
F_{1}<0 \text { or }\left(F_{2}<0 \text { and } F_{2}^{2}>3 F_{1} F_{3}\right)
$$

Hence, 
Table 1. Values of $F_{i}(i=0,1,2,3)$ of the coefficients $b_{0}\left(k^{2}\right)$ and $b_{1}\left(k^{2}\right) b_{2}\left(k^{2}\right)-b_{0}\left(k^{2}\right)$ which are used to determine conditions for Turing instability.

\begin{tabular}{|c|c|c|}
\hline & $b_{0}$ & $b_{1} b_{2}-b_{0}$ \\
\hline$F_{3}$ & $d_{1} d_{3}$ & $\left(1+d_{1}\right)\left(1+d_{3}\right)\left(d_{1}+d_{3}\right)$ \\
\hline & & $-\left(1+d_{3}\right)\left(1+2 d_{1}+d_{3}\right) a_{11}$ \\
\hline$F_{2}$ & $d_{1} a_{33}+d_{3} a_{11}+d_{1} d_{3} a_{22}$ & $\begin{array}{l}-\left(d_{1}+d_{3}\right)\left(2+d_{1}+d_{3}\right) a_{22} \\
-\left(1+d_{1}\right)\left(1+d_{1}+2 d_{3}\right) a_{33}\end{array}$ \\
\hline$F_{1}$ & $\begin{array}{l}d_{1}\left(a_{22} a_{33}-a_{23} a_{32}\right)+a_{11} a_{33} \\
+d_{3}\left(a_{11} a_{22}-a_{12} a_{21}\right)\end{array}$ & $\begin{array}{l}\left(1+d_{1}\right)\left(a_{33}^{2}-a_{12} a_{21}\right)+\left(d_{1}+d_{3}\right) a_{22}^{2} \\
+\left(1+d_{3}\right)\left(a_{11}^{2}-a_{23} a_{32}\right) \\
+2\left(1+d_{1} d_{3}\right)\left(a_{22} a_{33}+a_{11} a_{22}+a_{11} a_{33}\right)\end{array}$ \\
\hline$F_{0}$ & $b_{0}(0)$ & $b_{1}(0) b_{2}(0)-b_{0}(0)$ \\
\hline
\end{tabular}

$$
\begin{aligned}
F_{\min } & =F\left(k_{c}^{2}\right) \\
& =\frac{2 F_{2}^{3}-9 F_{1} F_{2} F_{3}-2\left(F_{2}^{2}-3 F_{1} F_{3}\right)^{3 / 2}+27 F_{3}^{2} F_{0}}{27 F_{3}^{2}}
\end{aligned}
$$

Thus,

$F\left(k_{c}^{2}\right)<0$

if $2 F_{2}^{3}-9 F_{1} F_{2} F_{3}-2\left(F_{2}^{2}-3 F_{1} F_{3}\right)^{3 / 2}+27 F_{3}^{2} F_{0}<0$

The conditions given in Equations (9) and (11) are sufficient for the occurrence of Turing instability.

At bifurcation, when $F_{\min }=0$, we require

$$
2 F_{2}^{3}-9 F_{1} F_{2} F_{3}-2\left(F_{2}^{2}-3 F_{1} F_{3}\right)^{3 / 2}+27 F_{3}^{2} F_{0}=0
$$

The above discussion is summarized in the following theorem.

Theorem 3.1: The spatio-temporal system (2) will undergo Turing instability at the homogeneous steady state $E$ provided the following two conditions are satisfied:

1) $F_{1}<0$ or $\left(F_{2}<0\right.$ and $\left.F_{2}^{2}>3 F_{1} F_{3}\right)$

2) $2 F_{2}^{3}-9 F_{1} F_{2} F_{3}-2\left(F_{2}^{2}-3 F_{1} F_{3}\right)^{3 / 2}+27 F_{3}^{2} F_{0}<0$

Proof: The proof directly follows from the above discussion.

Based on the linear stability analysis of system (2), a two-parameter bifurcation diagram with respect to parameters $c_{1}$ and $d_{1}$ is obtained for the parametric choice

$$
c_{2}=0.9, c_{3}=0.4, c_{4}=0.1, c_{5}=0.3, c_{6}=0.15, d_{3}=10 .
$$

For this parametric choice, the Hopf bifurcation point is computed as $c_{1}=1.26882$ and the expression for the Turing bifurcation curve is obtained by solving Equation (3.10) for $c_{1}$, as a function of $d_{1}$. The Hopf line and the Turing bifurcation curve, which are shown in the bifurcation diagram Figure 1, separate the parametric space

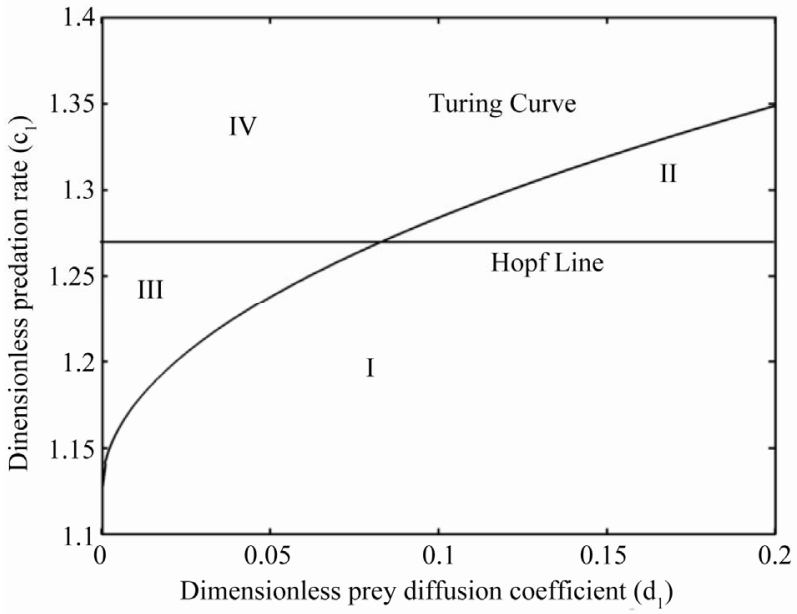

Figure 1. Two parameter bifurcation diagram for the system (2) with parameters (13).

in to four distinct domains. In domain I, located below both of the lines, the steady state is the only stable solution of the system. Domain II and III are the regions of pure Hopf and pure Turing instabilities respectively, while domain IV, which is located above both the curves, is the region of both Hopf and Turing instabilities.

For Turing instability to occur, at least one of the coefficients of the dispersion relation must be negative for some range of $k^{2}$. From Figure 2 it is clear that the coefficient $b_{0}\left(k^{2}\right)$ is negative in the range $0<k^{2}<6$.

\section{Spatio-Temporal Pattern Formation}

It is well known that it is not always possible to obtain the analytical solutions of coupled system of nonlinear PDE. Hence, one has to use numerical simulations to solve them. The spatio-temporal system (2) is solved numerically in two-dimensional space using a finite difference approximation for the spatial derivatives and an explicit Euler method for the time integration [11]. In order to avoid numerical artifacts the values of the time and space steps have been chosen sufficiently small. This method finally results to a sparse, banded linear system of algebraic equations. The linear system obtained is then solved by using GMRES algorithm [11].

For the numerical simulations, the initial distributions of the species are considered as small spatial perturbation of the uniform equilibrium point.

All the numerical simulations employ the zero-flux (Neumann) boundary conditions in a square habitat of size $50 \times 50$. Iterations are performed for different step sizes in time and space until the solution seems to be invariant. The time step size of $\Delta t=0.01$ and space step size $\Delta x=\Delta y=0.25$ are chosen. In this section, extensive numerical simulations of the spatially extended 


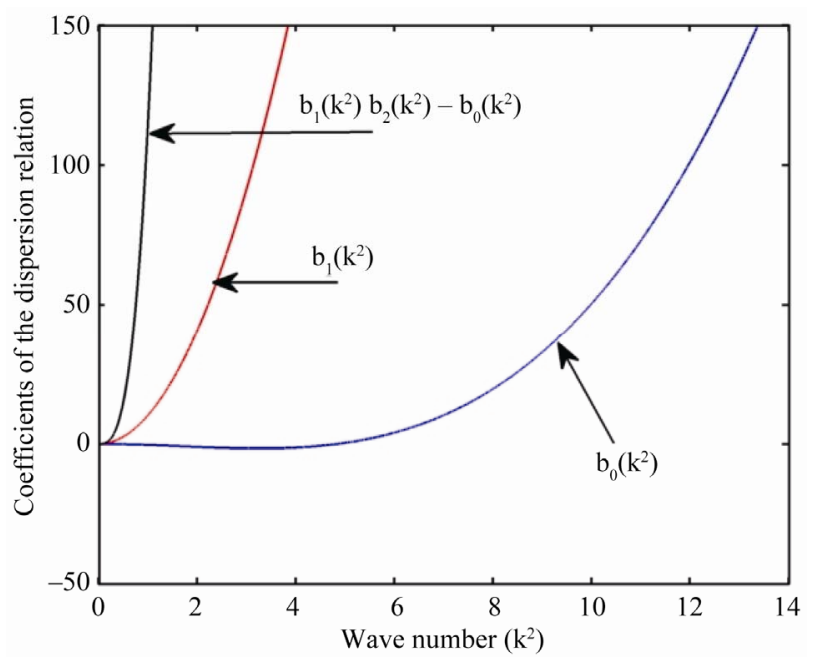

Figure 2. Coefficient of the dispersion relation (5) of the system (2) for parameters (13), $c_{1}=1.24, d_{1}=0.01$.

system (2) in two-dimensional space are performed and the qualitative results are analyzed. The following system parameters are chosen as fixed based on the stability and bifurcation analysis carried out in Section 3, whereas the control parameter $c_{1}$ is varied in the simulation experiments:

$$
\begin{aligned}
& c_{2}=0.9, c_{3}=0.4, c_{4}=0.1, c_{5}=0.3, c_{6}=0.15, \\
& d_{1}=0.01, d_{3}=10 .
\end{aligned}
$$

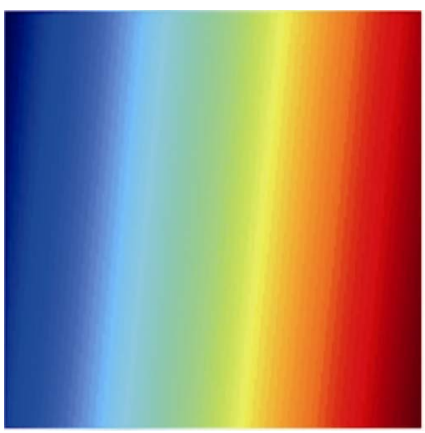

(a)

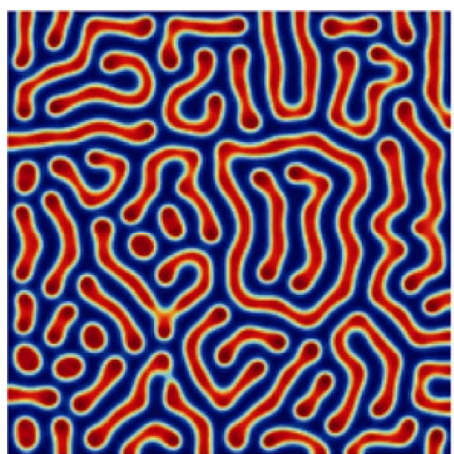

(c)
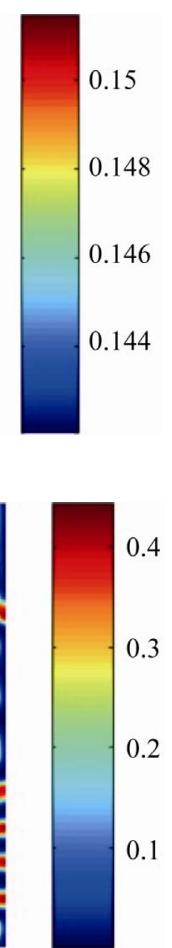

\section{2}

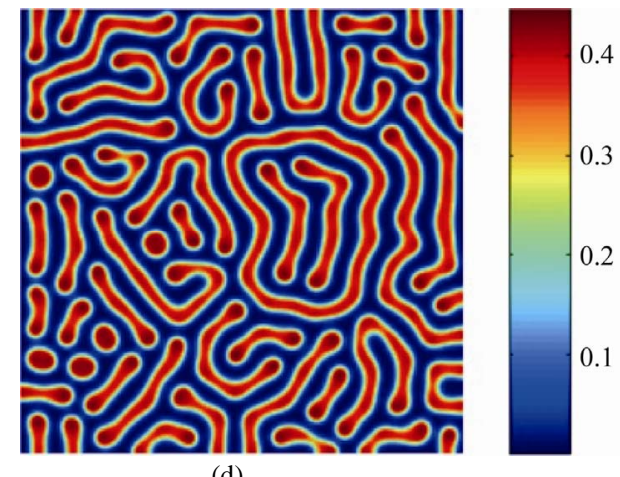

(d)

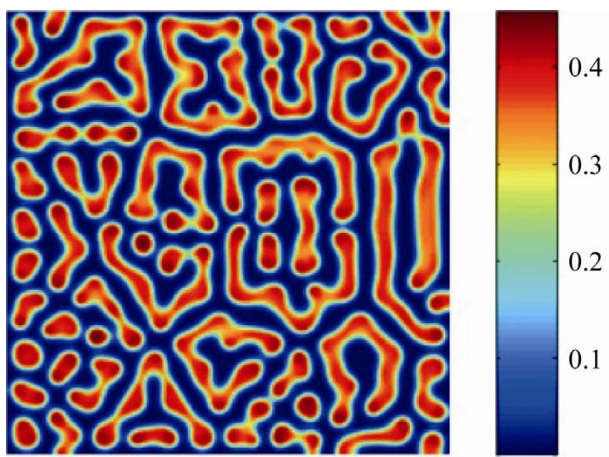

(b)

From the analysis and phase-transition bifurcation diagram of Figure 1, it is observed that the system dynamics is determined by the values of $c_{1}$ and $d_{1}$. For different sets of parameters, the feature of the spatial patterns become essentially different if $c_{1}$ exceeds the Hopf bifurcation threshold $c_{1 \text { Hopf }}$ and Turing bifurcation threshold $c_{1 \text { Turing }}$, which depends on $d_{1}$, respectively. For the choice of parameters given in (14), Turing bifurcation threshold and Hopf bifurcation threshold are computed as $c_{1 \text { Turing }}=1.1753$ and $c_{1 \text { Hopf }}=1.26882$ respectively.

For $c_{1}=1.35$, which is greater than both the Turing bifurcation threshold and the Hopf bifurcation threshold, the system parameters lie in domain IV of the bifurcation diagram of Figure 1.

In two species spatial models, the patterns of the prey and the predator are of the same type. As a result, the analysis of pattern formation can be restricted to one of the species only [17-19]. However, different behavior is observed for the three species system. Accordingly the Figures 3-5 are drawn for prey, intermediate predator and top predator respectively for $c_{1}=1.35$. In each of these figures, slide (a) corresponds to the initial distribution in the habitat. The time evolution of the patterns at 50000, 200000 and 500000 iterations are shown in slides (b), (c) and (d), respectively. The coexistence of spotted pattern, ring-shaped and the stripe like patterns are observed for the three species.

Figure 3. Patterns of the prey for $c_{1}=1.35$ at different time steps (iterations) (a) 0 ; (b) 50,000; (c) 200,000; (d) 500,000. 


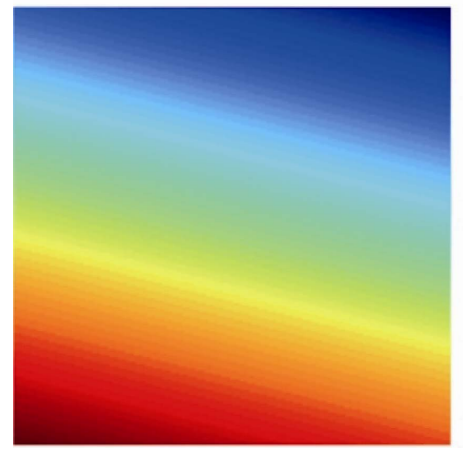

(a)

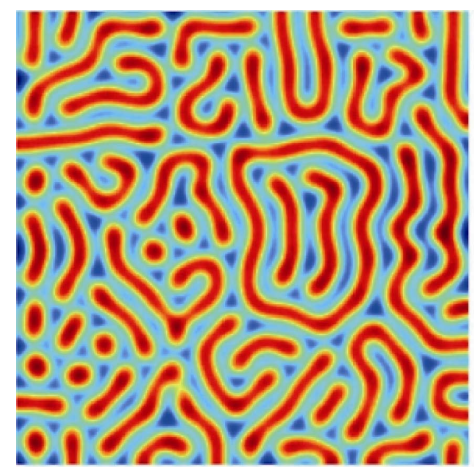

(c)
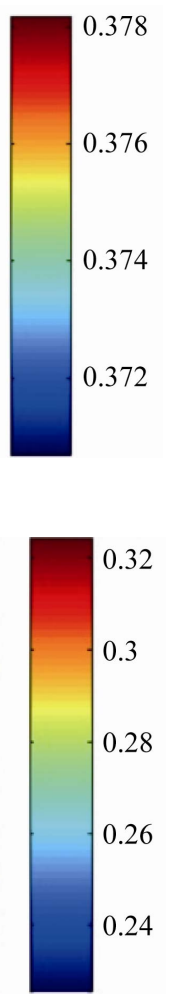

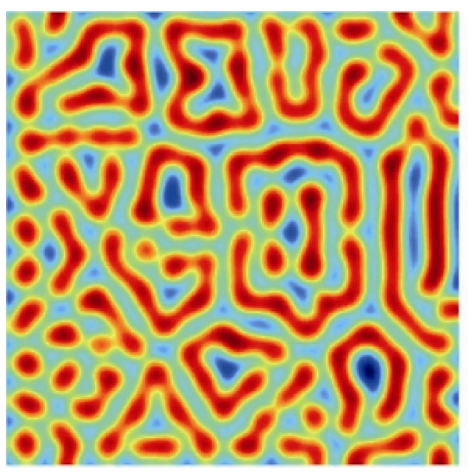

(b)

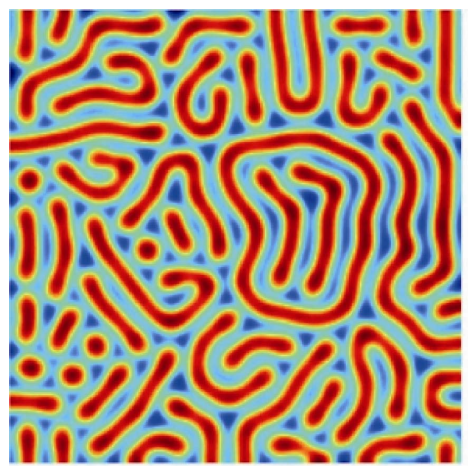

(d)
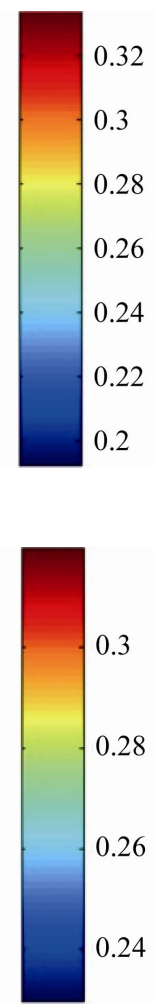

0.24

Figure 4. Patterns of the intermediate predator for $c_{1}=1.35$ at different time steps (iterations) (a) 0 ; (b) 50,000; (c) 200,000; (d) 500,000.

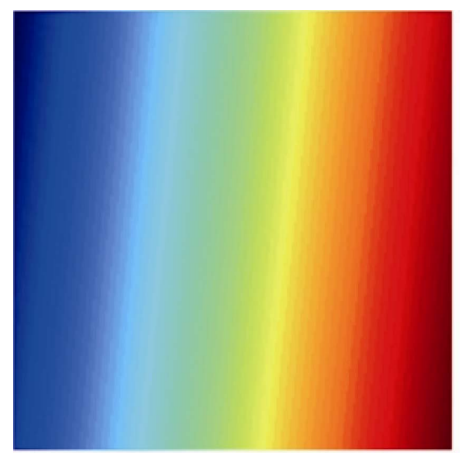

(a)

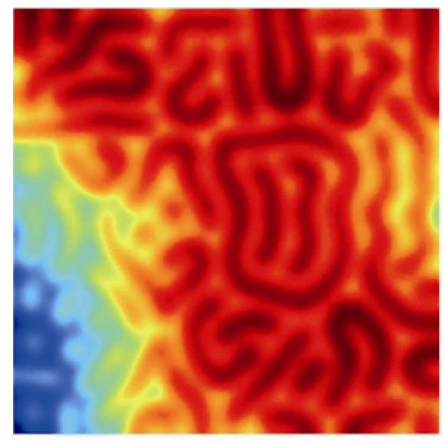

(c)
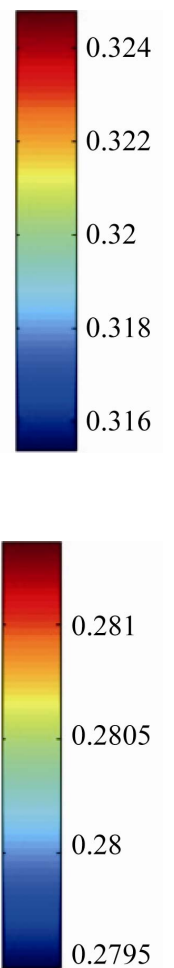

0.2795

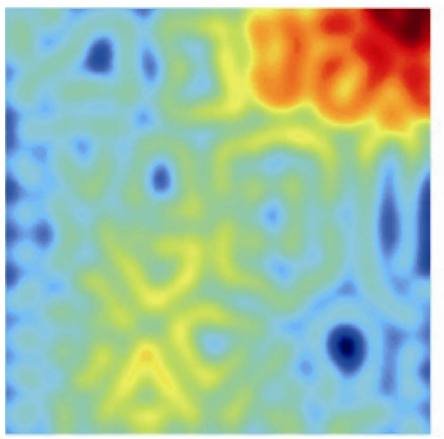

(b)

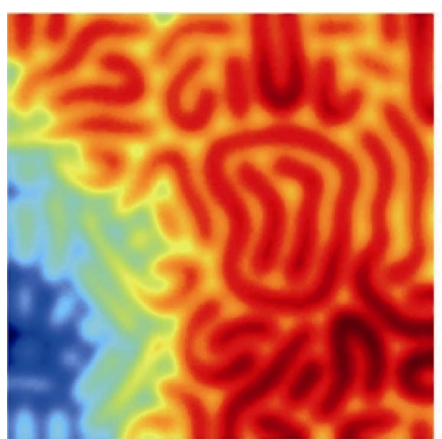

(d)
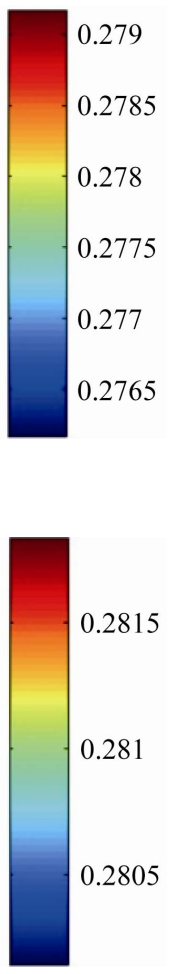

Figure 5. Patterns of the top predator for $c_{1}=1.35$ at different time steps (iterations) (a) 0; (b) 50,000; (c) 200,000; (d) 500,000. 
In Figure 3, one can see that the small spatial perturbbations to the homogeneous steady state of the spatiotemporal system (2) leads to the formation of spots and stripes of high prey density on a blue background of low prey density (c.f. Figure 3(b)). However, at later time some of the spots merge together to form stripes. This results in an increase in the number of stripes and a decrease in the number of spots. Ring-shaped pattern is also formed (c.f. Figures 3(c) and (d)).

The patterns of the intermediate predator shown in Figure $\mathbf{4}$ is structurally similar with that of the patterns of the prey shown in Figure 3. But, the background color is not spatially uniform and varying temporally. In particular, the background density is decreasing with time.

The basic skeleton of the pattern of the top predator (c.f. Figure 5) is similar with that of the patterns of the prey and intermediate predator. However the background density and the density in the patterns keep changing spatially as well as temporally. Patches of different densities are also visible.

Figure 6 shows the steady state patterns of the three species for $c_{1}=1.24$. In this case, the control parameter $c_{1}$ is greater than the Turing bifurcation threshold $c_{1 \text { Turing }}$ but less than the Hopf bifurcation threshold $c_{1 \text { Hopf }}$. That is, the parametric space is domain III of the bifurcation diagram of Figure 1. This means that the system has pure Turing instability and Turing patterns are expected for this choice. The numerical simulation

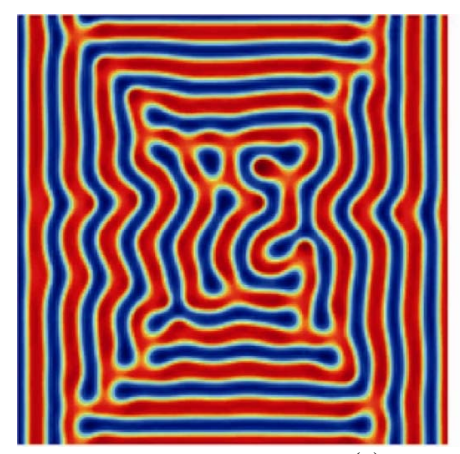

(a)

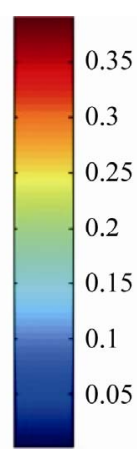

0.05

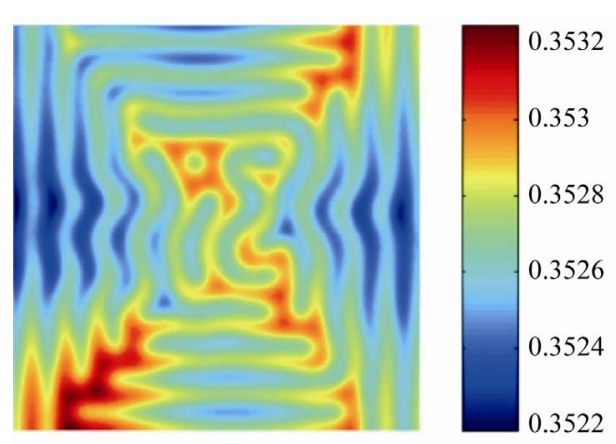

(c)

Figure 6. Steady state patterns of the prey (a), intermediate predator (b) and top predator (c) for $c_{1}=1.24$. 
be useful for studying the dynamic complexity of ecosystems or physical systems.

\section{References}

[1] A. Al-Khedhairi, "The Chaos and Control of Food Chain Model Using Nonlinear Feedback," Applied Mathematical Sciences, Vol. 3, No. 12, 2009, pp. 591-604.

[2] N. J. Gotelli and A. M. Ellison, "Food-Web Models Predict Species Abundances in Response to Habitat Change," PLoS Biology, Vol. 4, No. 10, 2006, pp. 1869-1873. doi:10.1371/journal.pbio.0040324

[3] S. Gakkhar and R. K. Naji, "Chaos in Three Species Ratio Dependent Food Chain,” Chaos, Solitons and Fractals, Vol. 14, No. 5, 2002, pp. 771-778. doi:10.1016/S0960-0779(02)00038-3

[4] S. B. Hsu, T. W. Hwang and Y. Kuang, "A RatioDependent Food Chain Model and Its Applications to Biological Control,” Mathematical Biosciences, Vol. 181, No. 1, 2003, pp. 55-83. doi:10.1016/S0025-5564(02)00127-X

[5] K. A. J. White and C. A. Gilligan, "Spatial Heterogeneity in Three-Species, Plant-Parasite-Hyperparasite, Systems," Philosophical Transactions of Royal Society B, Vol. 353, No. 1368, 1998, pp. 543-557. doi:10.1098/rstb.1998.0226

[6] C. Neuhauser and S. W. Pacala, "An Explicitly Spatial Version of Lotka-Voltera Model with Inter-Specific Competition,” The Annals of Applied Probability, Vol. 9, No. 4, 1999, pp. 1226-1259. doi:10.1214/aoap/1029962871

[7] K. S. McCann, J. B. Rasmussen and J. Umbanhowar, “The Dynamics of Spatially Coupled Food Webs," Ecology Letters, Vol. 8, No. 5, 2005, pp. 513-523. doi:10.1111/j.1461-0248.2005.00742.X

[8] S. H. Lee, H. K. Pak, H. S. Wi, T. S. Chon and T. Matsumoto, "Growth Dynamics of Domain Pattern in a Three-Trophic Population Model,” Physica A, Vol. 334, No. 1-2, 2004, pp. 233-242. doi:10.1016/j.physa.2003.11.017

[9] D. O. Maionchi, S. F. dos Reis and M. A. M. de Aguiar, "Chaos and Pattern Formation in a Spatial Tritrophic Food Chain,” Ecological Modelling, Vol. 191, No. 2, 2006, pp. 291-303. doi:10.1016/j.ecolmodel.2005.04.028

[10] M. Wang, "Stationary Patterns for a Prey-Predator Model with Prey-Dependent and Ratio-Dependent Functional Responses and Diffusion,” Physica D, Vol. 196, No. 1-2, 2004, pp. 172-192. doi:10.1016/j.physd.2004.05.007

[11] M. R. Garvie, "Finite-Difference Schemes for ReactionDiffusion Equations Modeling Predator-Prey Interactions in MATLAB,” Bulletin of Mathematical Biology, Vol. 69, No. 3, 2007, pp. 931-956. doi:10.1007/s11538-006-9062-3

[12] A. B. Medvinsky, S. V. Petrovskii, I. A. Tikhonov, H. Malchow and B. L. Li, "Spatiotemporal Complexity of
Plankton and Fish Dynamics,” SIAM Review, Vol. 44, No. 3, 2002, pp. 311-370. doi:10.1137/S0036144502404442

[13] S. V. Petrovskii, B. L. Li and H. Malchow, "Transition to Spatiotemporal Chaos Can Resolve the Paradox of Enrichment,” Ecological Complexity, Vol. 1, No. 1, 2004, pp. 37-47. doi:10.1016/j.ecocom.2003.10.001

[14] H. Shen and Z. Jin, "Two Dimensional Pattern Formation of Prey-Predator System,” Eighth ACIS International Conference on Software Engineering, Artificial Intelligence, Networking, and Parallel/Distributed Computing, Qingdao, 30 July - 1 August 2007, pp. 343-346. doi:10.1109/SNPD.2007.215

[15] A. M. Turing, "The Chemical Basis of Morphogenesis," Philosophical Transactions of Royal Society B, Vol. 237, No. 641, 1952, pp. 37-72. doi:10.1098/rstb.1952.0012

[16] M. Banerjee and S.V. Petrovskii, "Self-Organized Spatial Patterns and Chaos in a Ratio-Dependent Predator Prey System,” Theoretical Ecology, Vol. 4, No. 1, 2011, pp. 37-53. doi:10.1007/s12080-010-0073-1

[17] W. Wang, L. Zhang, Y. Xue and Z. Jin, "Spatiotemporal Pattern Formation of Beddington-DeAngelis-Type Predator-Prey Model,” arXiv: 0801.0797v1 [q-bio.PE], January 2008.

[18] L. Zhang, W. Wang and Y. Xue, "Spatiotemporal Complexity of a Predator-Prey System with Constant Harvest Rate,” Chaos Solitons Fractals, Vol. 41, No. 1, 2009, pp. 38-46. doi:10.1016/j.chaos.2007.11.009

[19] W. Wang, Q. X. Liu and Z. Jin, "Spatiotemporal Complexity of a Ratio-Dependent Predator-Prey System,” Physical Review E, Vol. 75, No. 5, 2007, Article ID 051913. doi:10.1103/PhysRevE.75.051913

[20] J. D. Murray, "Mathematical Biology II: Spatial Models and Biomedical Applications,” Springer, Berlin, 2003.

[21] G. Sun, Z. Jin, Q. X. Liu and L. Li, "Pattern Formation in a Spatial S-I model with Nonlinear Incidence Rates," Journal of Statistical Mechanics: Theory and Experiment, Vol. 2007, 2007, P11011. doi:10.1088/1742-5468/2007/11/P11011

[22] H. Malchow, "Spatio-Temporal Pattern Formation in Coupled Models of Plankton Dynamics and Fish School Motion," Nonlinear Analysis: Real World Applications, Vol. 1, No. 1, 2000, pp. 53-67. doi:10.1016/S0362-546X(99)00393-4

[23] S. B. L. Araújo and M. A. M. de Aguiar, "Pattern Formation, Outbreaks, and Synchronization in Food Chains with Two and Three Species," Physical Review E, Vol. 75, No. 6, 2007, Article ID 061908. doi:10.1103/PhysRevE.75.061908

[24] W. Ko and I. Ahn, "Analysis of Ratio-Dependent Food Chain Model," Journal of Mathematical Analysis Applications, Vol. 335, No. 1, 2007, pp. 498-523. doi:10.1016/j.jmaa.2007.01.089 